\title{
MEASURABLE GAMBLING HOUSES
}

\author{
BY \\ RALPH E. STRAUCH
}

1. Introduction. In [4], Dubins and Savage develop a general theory of gambling. The gambler begins with an initial fortune $f$, which is a point in a fortune space $F$. He may either stop at $f$ or select a gamble $\gamma$, which is a probability on $F$, from a set $\Gamma(f)$ of gambles available at $f$. He then moves to a new fortune $f^{\prime}$ selected according to $\gamma$, where he repeats the process. A bounded utility function $u$ is defined on $F$, and the gambler's objective is to stop at a fortune of high utility. If we let $U(f)$ be the best he can expect to do starting at $f$, then we say that $U$ is the utility of the house. The theory uses finitely additive gambles defined on all subsets of $F$, partly because of measurability difficulties which arise if the usual measurability structure of countably additive gambles defined on a $\sigma$-field of subsets of $F$ is used. In particular, the utility of the house need not be measurable (see example below).

In this paper we show that given a Borel measurability structure of the type used by Blackwell in dynamic programming [1], the utility of the house, while not necessarily Borel measurable, is absolutely measurable and hence its integral is defined with respect to any Borel measure. (A function is absolutely measurable if it is measurable with respect to the completion of the Borel sets under any measure.) Moreover, the gambler can do as well using only measurable policies (defined below) as he can using arbitrary policies. The assumptions, and hence the results, are somewhat weaker than those of $\$ 2.16$ of [4]. The methods used are the same as those used in $\S 7$ of [8] to obtain similar results in dynamic programming.

$\S 4$ is somewhat of a digression and contains the following results: If $(\Omega, \mathscr{F})$ is a measurable space and $\mathscr{F}_{n}$ is an increasing sequence of countably generated sub- $\sigma$-fields of $\mathscr{F}$, then there exists a countable collection of stop rules $C$ such that for any stop rule $t$ and any probability $p$ on $(\Omega, \mathscr{F})$, there is a sequence of stop rules $\left\{t_{m}\right\} \subset C$ such that $t_{m} \rightarrow t$ with $p$-probability one.

2. The gambler's problem. In this section we outline the gambler's problem as defined in Chapter 2 of [4]. The necessary theory of finitely additive integration in product spaces is developed there. We will make no use of that theory in this paper.

Let $F$ be some nonempty set consisting of fortunes $f$. A gamble $\gamma$ is a finitely additive probability defined on all subsets of $F$, and a gambling house $\Gamma$ is a function which associates with each fortune $f$ a nonempty set $\Gamma(f)$ of gambles $\gamma$ among

Received by the editors November 18, 1965. 
which the gambler is allowed to choose when his fortune is $f$. We refer to $\Gamma(f)$ as the set of gambles available in $\Gamma$ at $f$.

A strategy $\sigma$ is a sequence $\sigma_{0}, \sigma_{1}, \ldots$, where $\sigma_{0}$ is a gamble and for each $n>0$, $\sigma_{n}$ is a function which associates, with each finite sequence of fortunes $\left(f_{1}, \ldots, f_{n}\right)$, a gamble $\sigma_{n}\left(f_{1}, \ldots, f_{n}\right)$. We think of $\sigma_{n}\left(f_{1}, \ldots, f_{n}\right)$ as the conditional distribution of $f_{n+1}$ if the gambler has experienced $f_{1}, \ldots, f_{n}$ and uses the strategy $\sigma$. The strategy $\sigma$ induces a probability on the space of histories $H=F \times F \times \cdots$, which we shall also denote by $\sigma$. The strategy $\sigma$ is available in $\Gamma$ at $f$ if $\sigma_{0} \in \Gamma(f)$ and $\sigma_{n}\left(f_{1}, \ldots, f_{n}\right)$ $\in \Gamma\left(f_{n}\right)$ for $n \geqq 1$.

A stop rule $t$ is an integer-valued function or the set of histories $H$ with the property that if $h$ and $h^{\prime}$ agree through the first $t(h)$ coordinates, then $t(h)=t\left(h^{\prime}\right)$. Associated with each stop rule $t$ is the terminal fortune $f_{t}$, the fortune at which we stop using $t$. A policy $\pi$ is a pair $(\sigma, t) ; \pi$ is available in $\Gamma$ at $f$ if $\sigma$ is available at $f$.

The utility function $u$ is a bounded real-valued function on $F$, and $u(f)$ is the utility of the fortune $f$ to the gambler. If the gambler uses the policy $\pi=(\sigma, t)$, i.e., gambles according to $\sigma$ until time $t$ and then stops, his utility when he stops will be $u\left(f_{t}\right)$. The utility $u(\pi)$ of the policy $\pi$ is the expected utility on stopping, i.e.,

$$
u(\pi)=\int u\left(f_{t}\right) d \sigma
$$

The gambler wishes to find a policy $\pi$ which will make $u(\pi)$ as large as possible, given his initial fortune $f$ and the rules of the house $\Gamma$, or to stop immediately and settle for $u(f)$ if he finds that preferable. He then wishes to achieve, or at least to approach,

$$
U(f)=\max [u(f), \sup u(\pi)],
$$

where the supremum is taken over those policies $\pi$ available in $\Gamma$ at $f$. We call $U(f)$ the utility of $\Gamma$ at $f$.

3. Measurable houses. Let $F$ be a nonempty Borel set, i.e., a Borel subset of a complete separable metric space, and let $\mathscr{B}$ be the $\sigma$-field of its Borel subsets. Let $P$ be the set of countably additive probabilities $p$ on $\mathscr{B}$, and let $\Sigma$ be the smallest $\sigma$-field of subsets of $P$ which makes $p B$ a $\Sigma$ measurable function of $p$ for each $B \in \mathscr{B}$. Then $P$ is a Borel set, and $\Sigma$ is the $\sigma$-field of its Borel subsets [3].

We will abuse notation somewhat by using $\gamma$ throughout the rest of the paper to denote the restriction of a gamble to $\mathscr{B}$, and will identify all gambles which agree on $\mathscr{B}$. If all gambles available in $\Gamma$ are countably additive, we will also let $\Gamma$ denote the set of pairs $\{(f, \gamma) \mid \gamma \in \Gamma(f)\}$ in the product space $F \times P$.

Definition 1. A gambling house $\Gamma$ is measurable if:

(i) The set of fortunes $F$ for which $\Gamma$ is defined is a Borel set.

(ii) Each gamble $\gamma$ available in $\Gamma$ is countably additive when restricted to $\mathscr{B}$.

(iii) $\Gamma=\{(f, \gamma) \mid \gamma \in \Gamma(f)\} \in \mathscr{B} \times \Sigma$.

Definition 2. A strategy $\sigma$ available in a measurable house $\Gamma$ is measurable if 
$\sigma_{n}$ is a measurable map from $F \times F \times \cdots \times F$ ( $n$ factors) to $P$, i.e., if $\sigma_{n}$ is a regular conditional probability on $(F, \mathscr{B})$ given $\left(f_{1}, \ldots, f_{n}\right)$.

Definition 3. A policy $\pi=(\sigma, t)$ is measurable if $\sigma$ is measurable and $t$ is measurable with respect to $\mathscr{B}^{\infty}$, the product $\sigma$-field in $H$.

The main result of this paper is

THEOREM 1. If $\Gamma$ is a measurable house and $u$ is Borel measurable, then $U$ is absolutely measurable, and for any $\varepsilon>0$, and any $f$ there exists a measurable policy $\pi$ available at $f$ such that $u(\pi)>U(f)-\varepsilon$.

The following example, which is closely related to Example 2 of [1], shows that under the conditions of the theorem, $U$ need not be Borel measurable.

EXAMPLE. Let $F$ be the unit square, and for $f=\left(x_{0}, y_{0}\right) \in F$, let

$$
\Gamma\left(x_{0}, y_{0}\right)=\left\{\delta\left(x_{0}, y\right) \mid 0 \leqq y \leqq 1\right\},
$$

where $\delta(x, y)$ is the measure which assigns probability one to the point $(x, y)$. Let $u$ be the indicator function of $B$, a Borel subset of $F$ whose projection $D$ on the $x$-axis is not Borel. Then $U(x, y)=1$ if $x \in D$ and $U(x, y)=0$ otherwise; hence $U$ is not Borel measurable.

Dubins and Savage also define the strategic utility of the house, $V$, as the best the gambler can expect to do if he must play an arbitrarily long time before stopping [4, Chapter 3]. We do not know if a similar result holds for $V$.

The remainder of the paper is devoted to the proof of Theorem 1.

4. A digression about stop rules. This section develops a result for stop rules which we will need for the proof of Theorem 1, but which is independent of the rest of the paper. We give the result in somewhat greater generality than it is needed in the paper, and for that reason the notation in this section is also independent of that in the rest of the paper.

Let $(\Omega, \mathscr{F})$ be a measurable space, i.e., let $\Omega$ be a set and $\mathscr{F}$ be a $\sigma$-field of subsets of $\Omega$, and let $\left\{\mathscr{F}_{n}, n \geqq 1\right\}$ be a nondecreasing sequence of countably generated sub- $\sigma$-fields of $\mathscr{F}$. Let $T$ be the collection of all stop rules $t$, i.e., integer-valued random variables $t$ such that $\{\omega \mid t(\omega) \leqq n\} \in \mathscr{F}_{n}$. Let $P$ be the set of all probability measures $p$ on $(\Omega, \mathscr{F})$.

THEOREM 2. There exists a countable subset $C \subset T$ such that for any $t \in T$ and any $p \in P$, there exists a sequence $\left\{t_{k}, k=1,2, \ldots\right\} \subset C$ such that

$$
\lim _{k \rightarrow \infty} t_{k}=t \quad \text { with p-probability one. }
$$

Proof. Let $\mathscr{F}_{n m}, n, m \geqq 1$, be finite fields such that $\mathscr{F}_{n m} \subset \mathscr{F}_{n^{\prime} m^{\prime}}$ for all $n \leqq n^{\prime}$ and $m \leqq m^{\prime}$, and the smallest $\sigma$-field containing $\bigcup_{m} \mathscr{F}_{n m}$ is $\mathscr{F}_{n}$. Let

$$
\begin{aligned}
C_{m} & =\left\{t \in T \mid t \leqq m \text { and }[t=n] \in \mathscr{F}_{n m} \text { for } 1 \leqq n \leqq m\right\}, \\
C & =\bigcup_{m=1}^{\infty} C_{m} .
\end{aligned}
$$


Then $C$ is countable, since each $C_{m}$ is finite, and we need only show that $C$ satisfies the conditions of the theorem.

Take any $t \in T$, and any $p \in P$. Let $A_{n}=\{\omega \mid t(\omega)=n\}$. For each $n$, we can find a sequence of sets $B_{n m}^{\prime}$ such that

$$
B_{n m}^{\prime} \in \mathscr{F}_{n m} \text { and } \lim _{m \rightarrow \infty} P\left(B_{n m}^{\prime} \Delta A_{n}\right)=0,
$$

where

$$
B \Delta A=[A \cap(\Omega-B)] \cup[B \cap(\Omega-A)] .
$$

(See [2, supplement Theorem 1.3].) Let

$$
\begin{aligned}
& B_{n m}=B_{n m}^{\prime}-\bigcup_{j<n} B_{j m}^{\prime} \quad \text { for } n<m, \\
& B_{m m}=\Omega-\bigcup_{j<m} B_{j m}^{\prime},
\end{aligned}
$$

and define $t_{m}$ by $B_{n m}=\left\{\omega \mid t_{m}(\omega)=n\right\}$.

Clearly $t_{m} \in C_{m}$ for all $m$, and routine calculations show that $t_{m} \rightarrow t$ in $p$-probability as $m \rightarrow \infty$; hence by a well-known theorem [6, p. 116], we can extract a subsequence which converges to $t$ with $p$-probability one.

COROLlary 1. If $Y_{n}$ is any sequence of uniformly bounded random variables on $(\Omega, \mathscr{F})$, then $\sup _{t \in T} \int Y_{t} d p$ is a $\Sigma$ measurable function of $p$.

Proof. If $t_{n} \rightarrow t$ almost surely [ $p$ ], then $Y_{t_{n}} \rightarrow Y_{t}$ almost surely [ $p$ ]. Hence from Theorem 1 and the Lebesgue dominated convergence theorem [6, p. 125], it follows that

$$
\sup _{t \in C} \int Y_{t} d p=\sup _{t \in T} \int Y_{t} d p
$$

for all $p \in P$. But by Theorem 2.2 of [3], $\int Y_{t} d p$ is a $\Sigma$ measurable function of $p$ for each $t$; hence so is

$$
\sup _{t \in C} \int Y_{t} d p
$$

5. Random strategies. Let $X=P \times F \times P \times F \times \cdots$, i.e., let $X$ be the set of histories of the gambler, including both his fortunes and the gambles used. Let $X_{n}=P \times F \times \cdots \times F(2 n$ factors) be his partial history up to his $n$th fortune. We will index the coordinates of $x \in X$ by $x=\left(\gamma_{0}, f_{1}, \gamma_{1}, f_{2}, \ldots\right)$ and the coordinates of $x_{n} \in X_{n}$ similarly. We equip $X$ and the $X_{n}$ 's with their product $\sigma$-fields of Borel sets.

Definition 4. A random strategy $\rho$ is a sequence of $\rho_{0}, \rho_{1}, \ldots$, where $\rho_{0}$ is a probability on $(P, \Sigma)$ and for $n \geqq 1, \rho_{n}$ is a regular conditional probability on $(P, \Sigma)$ given $X_{n}$. A random strategy $\rho$ is available in $\Gamma$ at $f$ if $\rho_{0}[\Gamma(f)]=1$, and for $n \geqq 1, \rho_{n}\left(\Gamma\left(f_{n}\right) \mid \gamma_{0}, f_{1}, \ldots, f_{n}\right)=1$.

The interpretation of a random strategy is that the gambler selects $\gamma_{0}$ according to $\rho_{0}$, and moves to $f_{1}$ according to $\gamma_{0}$. After experiencing the partial history 
$\left(\gamma_{0}, f_{1}, \ldots, f_{n}\right)$, he selects $\gamma_{n}$ according to $\rho_{n}$ and then moves to $f_{n+1}$ according to $\gamma_{n}$. It is clear that to any measurable strategy there corresponds a natural random strategy. The gambler gains nothing through the use of random strategies, as will be shown later. We introduce them only because they are needed in measurability arguments to follow.

Each random strategy $\rho$ induces in a natural way a probability, which we shall also denote by $\rho$, on the Borel subsets of $X$; i.e., $\rho$ is the probability such that the conditional distribution of $\gamma_{n}$ given $\left(\gamma_{0}, f_{1}, \ldots, f_{n}\right)$ is $\rho_{n}\left(\cdot \mid \gamma_{0}, f_{1}, \ldots, f_{n}\right)$ and the conditional distribution of $f_{n+1}$ given $\left(\gamma_{0}, f_{1}, \ldots, f_{n}, \gamma_{n}\right)$ is $\gamma_{n}$. Let $P^{*}$ be the set of probability measures $\mu$ on the Borel sets of $X$, and let $\Sigma^{*}$ be the smallest $\sigma$ field in $P^{*}$ which makes $\mu A$ a $\Sigma^{*}$ measurable function of $\mu$ for each Borel subset $A$ of $X$. Let

$$
R=\{(f, \mu) \mid \mu=\rho \text { for some } \rho \text { available in } \Gamma \text { at } f\} .
$$

\section{LEMMA 1. $R \in \mathscr{B} \times \Sigma^{*}$.}

Proof. $\mu=\rho$ for some $\rho$ available in $\Gamma$ at $f$ if and only if the conditional distribution of $f_{1}$ given $\gamma_{0}$ under $\mu$ is $\gamma_{0} \in \Gamma(f)$, and for $n \geqq 1$, the conditional distribution of $f_{n+1}$ given $\left(\gamma_{0}, \ldots, f_{n}, \gamma_{n}\right)$ is $\gamma_{n} \in \Gamma\left(f_{n}\right)$ almost surely with respect to $\mu$. The former condition is equivalent to, for all measurable functions $w$ on $X_{1}$,

$$
\int_{X_{1}} w\left(\gamma_{0}, f_{1}\right) d \mu\left(\gamma_{0}, f_{1}\right)=\int_{\left[\gamma_{0} \in \Gamma(f)\right]} \int w\left(\gamma_{0}, f_{1}\right) d \gamma_{0}\left(f_{1}\right) d \mu\left(\gamma_{0}\right)
$$

while the latter condition is equivalent to, for all $n \geqq 1$ and measurable functions $w$ on $X_{n+1}$,

$$
\begin{aligned}
\int_{X_{n+1}} w\left(\gamma_{0}, \ldots, f_{n}, \gamma_{n}, f_{n+1}\right) d \mu\left(\gamma_{0}, \ldots, f_{n+1}\right) \\
\quad=\int_{\left[\left(f_{n}, \gamma_{n}\right) \in \Gamma\right]} \int w\left(\gamma_{0}, \ldots, f_{n}, \gamma_{n}, f_{n+1}\right) d \gamma_{n}\left(f_{n+1}\right) d \mu\left(\gamma_{0}, \ldots, f_{n}, \gamma_{n}\right) .
\end{aligned}
$$

In the above integration, we use $\mu$ to denote its marginal on spaces of lower dimension, those spaces being indicated by the variables of integration.

For $n \geqq 0$, let $w_{n m}, m \geqq 1$, be a sequence of measurable functions on $X_{n+1}$ which separates probabilities, i.e., such that if $\mu \neq \nu$ on $X_{n+1}$, there exists a $j$ such that

$$
\int_{X_{n+1}} w_{n j} d \mu \neq \int_{X_{n+1}} w_{n j} d \nu
$$

Such a sequence exists. For example, we can let $w_{n m}, m \geqq 1$, be the indicator functions of sets in a countable field generating the Borel sets of $X_{n+1}$. For $m \geqq 1$, let

$$
R_{0 m}=\left\{(f, \mu) \mid(1) \text { holds for } w=w_{0 m}\right\}
$$


and for $n \geqq 1, m \geqq 1$, let

$$
R_{n m}=\left\{(f, \mu) \mid(2) \text { holds for } w=w_{n m}\right\} .
$$

Each integral defined in (1) and (2) is a Borel measurable function of $f$ and $\mu$ by Theorem 2.2 of [3]; hence each $R_{n m} \in \mathscr{B} \times \Sigma^{*}$, and

$$
R=\bigcap_{n=0}^{\infty} \bigcap_{m=1}^{\infty} R_{n m} .
$$

This completes the proof.

For any $\mu \in P^{*}$, and any measurable stop rule $t$, let

and let

$$
u(\mu, t)=\int u\left(f_{t}\right) d \mu
$$

$$
\begin{aligned}
u^{*}(\mu) & =\sup _{t} u(\mu, t), \\
Q(f) & =\max \left[u(f), \sup _{(f, \mu) \in R} u^{*}(\mu)\right] .
\end{aligned}
$$

(Any stop rule $t$ on $H$ determines in an obvious way a stop rule on $X$, which we shall also denote by $t$.)

We may interpret $u(\mu, t)$ as the gambler's utility if he were to stop a process with distribution $\mu$ using stop rule $t$, and $\mu^{*}(\mu)$ as the best he could do using measurable stop rules if the distribution of the process were $\mu$. If his initial fortune is $f$, he may choose the distribution of the process to be any $\mu$ with $(f, \mu) \in R$ or stop immediately; hence $Q(f)$ is the best he can do using random strategies and measurable stop rules.

LEMMA 2. $Q$ is absolutely measurable.

Proof. $u(\mu, t)$ is a $\Sigma^{*}$ measurable function of $\mu$ for each $t$. According to Corollary $1, u^{*}(\mu)$ is $\Sigma^{*}$ measurable. For each real $\lambda$,

$$
B_{\lambda}=\left\{(f, \mu) \mid(f, \mu) \in R, u^{*}(\mu)>\lambda\right\} \in \mathscr{B} \times \Sigma^{*} .
$$

Hence $C_{\lambda}=\{f \mid Q(f)>\lambda\}$, which is the projection of $B_{\lambda}$ on $F$, is analytic and so is absolutely measurable [5, p. 58].

6. Proof that $Q=U$. We defined $Q$ as the best the gambler could do using random strategies. We must now show first that he cannot do better with nonmeasurable strategies, and second that he can do as well with measurable (nonrandom) strategies.

LEMMA 3. $Q \geqq U$.

Proof. Clearly $Q \geqq u$; hence by Theorem 2.12 .1 of [4] we need only show that $Q$ is excessive for $\Gamma$, i.e., that for any $f_{0} \in F$ and any $\gamma \in \Gamma\left(f_{0}\right), \int Q d \gamma \leqq Q\left(f_{0}\right)$. 
Since $Q$ is absolutely measurable, there exists an $N_{1} \in \mathscr{B}$ such that $\gamma N_{1}=0$ and a $\mathscr{B}$ measurable $v$ such that for $f \notin N_{1}, v(f)=Q(f)$. Let

$$
A_{0}=\{f \mid v(f)=u(f)\},
$$

and for an arbitrary $\varepsilon>0$, let

$$
R_{\varepsilon}=\left\{(f, \mu) \in R \mid f \in N_{1} \cup A_{0}\right\} \cup\left\{(f, \mu) \in R \mid v(f)>u^{*}(\mu)-\varepsilon\right\} .
$$

Then $A_{0} \in \mathscr{B}, R_{\varepsilon} \in \mathscr{B} \times \Sigma^{*}$, and for each $f \in F$, the $f$ section of $R_{\varepsilon}$ is nonempty. Thus by Theorem 6.3 of [7], we can find a measurable map $\varphi$ from $F$ to $P^{*}$ and $N_{2} \in \mathscr{B}$ with $\gamma N_{2}=0$ such that for $f \notin N_{2}, \varphi(f) \in R_{\varepsilon}$. Let $\rho$ be the random strategy which uses $\gamma$ as the initial gamble and then uses $\varphi(f)$ if the resulting state is $f$, i.e.,

$$
\begin{array}{rlrl}
\rho_{0}=\delta(\gamma), & & \\
\rho_{n}\left(\gamma, f_{1}, \gamma_{1}, \ldots, f_{n}\right) & =\left[\varphi\left(f_{1}\right)\right]_{n-1}\left(\gamma_{1}, \ldots, f_{n}\right) & & \text { for } f_{1} \notin N \\
& =\rho_{n}^{\prime} & & \text { for } f_{1} \in N,
\end{array}
$$

where $\rho^{\prime}$ is some arbitrary strategy and $N=N_{1} \cup N_{2}$.

Let $\left\{t_{n}\right\}=C$ be a sequence of stop rules constructed as in $\S 2$, and let

$$
\begin{array}{r}
A_{n}=\left\{f \notin A_{0} \cup N \mid u\left(\varphi(f), t_{j}\right) \leqq u^{*}(\varphi(f))-\varepsilon \text { for } 1 \leqq j<n\right. \\
\text { and } \left.u\left(\varphi(f), t_{n}\right)>\mu^{*}(\varphi(f))-\varepsilon\right\} .
\end{array}
$$

Let $t$ be the stop rule defined by

$$
\begin{aligned}
t\left(f_{1}, f_{2}, \ldots\right) & =1 \quad \text { if } f_{1} \in A_{0} \cup N \\
& =t_{n}\left(f_{2}, f_{3}, \ldots\right)+1 \quad \text { if } f_{1} \in A_{n} \text { for } n \geqq 1 .
\end{aligned}
$$

Then

$$
\begin{aligned}
Q\left(f_{0}\right) & \geqq u(\rho, t)=\int_{A_{0}} u\left(f_{1}\right) d \gamma\left(f_{1}\right)+\sum_{n=1}^{\infty} \int_{A_{n}} u\left(\varphi\left(f_{1}\right), t_{n}\right) d \gamma\left(f_{1}\right) \\
& >\int Q\left(f_{1}\right) d \gamma\left(f_{1}\right)-\varepsilon .
\end{aligned}
$$

Since $\varepsilon$ was arbitrary, this completes the proof of Lemma 3. We now must show that the gambler gains nothing through the use of random strategies.

THEOREM 3. Let $\rho$ be any random strategy and $t$ be any stop rule. Then there exists a measurable strategy $\sigma$ such that $u(\sigma, t) \geqq u(\rho, t)$.

Proof. Let

$$
v_{0}\left(\gamma_{0}\right)=\int f_{t}(x) d \rho\left(x \mid \gamma_{0}\right)
$$


be the conditional expected utility of the policy $(\rho, t)$ after the gambler has choosen the initial gamble $\gamma_{0}$, and for $n \geqq 1$, let

$$
v_{n}\left(x_{n}, \gamma_{n}\right)=\int f_{t}(x) d \rho\left(x \mid x_{n}, \gamma_{n}\right)
$$

be the conditional expected utility of the policy $(\rho, t)$ after the gambler has experienced the partial history $x_{n}$ and chosen the gamble $\gamma_{n}$. (If he has already stopped, i.e., if $t(x) \leqq n$, then $v_{n}\left(x_{n}, \gamma_{n}\right)=f_{t}$.) Then

$$
\begin{aligned}
u(\rho, t) & =\int v_{0}\left(\gamma_{0}\right) d \rho_{0}\left(\gamma_{0}\right) \\
& =\iint v_{n}\left(x_{n}, \gamma_{n}\right) d \rho_{n}\left(\gamma_{n} \mid x_{n}\right) d \rho\left(x_{n}\right) \quad \text { for } n \geqq 1 ;
\end{aligned}
$$

i.e., the expected utility of $(\rho, t)$ is the expected value of the conditional expected utility at time $n$ for any $n$. We can find $\sigma_{0}^{\prime} \in \Gamma\left(f_{0}\right)$ such that

$$
v_{0}\left(\sigma_{0}^{\prime}\right) \geqq \int v_{0}\left(\gamma_{0}\right) d \rho_{0}\left(\gamma_{0}\right)
$$

and for $n \geqq 1$, by Lemma 2 of [1], we can find a measurable map $\sigma_{n}^{\prime}$ from $X_{n}$ to $P$ such that $\sigma_{n}^{\prime}\left(\gamma_{0}, f_{1}, \ldots, f_{n}\right) \in \Gamma\left(f_{n}\right)$ and

$$
v_{n}\left(x_{n}, \sigma_{n}^{\prime}\left(x_{n}\right)\right) \geqq \int v_{n}\left(x_{n}, \gamma_{n}\right) d \rho_{n}\left(\gamma_{n} \mid x_{n}\right) .
$$

Let $\rho^{(n)}=\delta\left(\sigma_{0}^{\prime}\right), \ldots, \delta\left(\sigma_{n-1}^{\prime}\right), \rho_{n}, \rho_{n+1}, \ldots$, be the strategy which uses $\sigma^{\prime}$ for the first $n$ gambles and then switches to $\rho$. An easy induction argument shows that

$$
u(\rho, t) \leqq u\left(\rho^{(1)}, t\right) \leqq \cdots
$$

Furthermore, since $u$ is bounded and all the probabilities involved are countably additive,

$$
\lim _{n \rightarrow \infty} u\left(\rho^{(n)}, t\right)=u\left(\sigma^{\prime}, t\right) .
$$

In the sense of the definition, $\sigma^{\prime}$ is not a measurable strategy, since $\sigma_{n}$ depends on past gambles as well as past fortunes. However, if we define the measurable strategy $\sigma$ by

$$
\begin{aligned}
& \sigma_{0}=\sigma_{0}^{\prime}, \\
& \sigma_{n}\left(f_{1}, \ldots, f_{n}\right)=\sigma_{n}^{\prime}\left(\sigma_{0}, f_{1}, \gamma_{1}, \ldots, \gamma_{n-1}, f_{n}\right),
\end{aligned}
$$

where $\gamma_{j}=\sigma_{j}^{\prime}\left(\sigma_{0}, f_{1}, \gamma_{1}, \ldots, f_{j}\right)$ for $j=1,2, \ldots, n-1$, then $\sigma^{\prime}$ and $\sigma$ induce the same probability on the spaces of histories $H$ and $X$; hence $u\left(\sigma^{\prime}, t\right)=u(\sigma, t)$. This completes the proofs of Theorems 1 and 3 . 


\section{REFERENCES}

1. David Blackwell, Discounted dynamic programming, Ann. Math. Statist. 36 (1965), 226-235.

2. J. L. Doob, Stochastic processes, Wiley, New York, 1953.

3. Lester Dubins and David Freedman, Measurable sets of measures, Pacific J. Math. 14 (1964), 1211-1222.

4. Lester E. Dubins and Leonard J. Savage, How to gamble if you must, McGraw-Hill, New York, 1965.

5 Casimir Kuratowski, Topologie I, Warsaw, Poland, 1933.

6. Michel Loeve, Probability theory, 2nd ed., Van Nostrand, Princeton, N. J., 1960.

7. George W. Mackey, Borel structure in groups and their duals, Trans. Amer. Math. Soc. 85 (1957), 134-165.

8. Ralph E. Strauch, Negative dynamic programming, Ann. Math. Statist. 37 (1966), 871-890.

THE RAND CORPORATION,

Santa Monica, California 